\title{
Research on Adaptive Threshold of Received Signal in Communication System
}

\author{
Xizheng Ke1,2, Xukuan Ji ${ }^{*}$ \\ ${ }^{1}$ Faculty of Automation and Information Engineering, Xi'an University of Technology, Xi'an, China \\ ${ }^{2}$ Shaanxi Civil-Military Integration Key Laboratory of Intelligence Collaborative Networks, Xi'an, China \\ Email: ^273796150@qq.com
}

How to cite this paper: Ke, X.Z. and Ji, X.K. (2021) Research on Adaptive Threshold of Received Signal in Communication System. Optics and Photonics Journal, 11, 1-11.

https://doi.org/10.4236/opj.2021.111001

Received: December 3, 2020

Accepted: January 19, 2021

Published: January 22, 2021

Copyright $\odot 2021$ by author(s) and Scientific Research Publishing Inc. This work is licensed under the Creative Commons Attribution International License (CC BY 4.0).

http://creativecommons.org/licenses/by/4.0/

\begin{abstract}
When the light beam propagates in the atmosphere, the signal will be absorbed and scattered by the gas molecules and water mist in the atmosphere, which will cause the loss of power rate. The complex atmospheric environment will produce a variety of adverse effects on the signal. The interference produced by these effects overlaps with each other, which will seriously affect the strength of the received signal. Therefore, how to effectively suppress the atmospheric turbulence effect in the random atmospheric turbulence channel, ensure the normal transmission of the signal in the atmospheric channel, and reduce the bit error rate of the communication system, is very necessary to improve the communication system. When processing the received signal, it is an important step to detect the transmitted signal by comparing the received signal with the threshold. In this paper, based on the atmospheric turbulence distribution model, the adaptive signal decision threshold is obtained through the estimation of high-order cumulant. Monte Carlo method is used to verify the performance of adaptive threshold detection. The simulation results show that the high-order cumulant estimation of atmospheric turbulence parameters can realize the adaptive change of the decision threshold with the channel condition. It is shown that the adaptive threshold detection can effectively restrain atmospheric turbulence, improve the performance of free space optical and improve the communication quality.
\end{abstract}

\section{Keywords}

Free Space Optical, Adaptive Threshold Detection, Higller-Order Cumulants

\section{Introduction}

Atmospheric laser communication, also known as free space optical (FSO), is a 
communication system that takes atmospheric channel as the transmission medium. Wireless optical communication has the characteristics of optical fiber communication and other wireless communication, and can realize the communication system with high bandwidth, low cost, convenient assembly and other capabilities. The power attenuation of the optical signal emitted by atmospheric laser communication system after passing through atmospheric turbulent channel results in the degradation of communication performance [1]. When processing received signals, threshold detection is particularly important, which can achieve a significant improvement in communication performance at a relatively low cost [2]. However, in the actual transmission, when the laser beam passes through the atmosphere, there must be a variety of noises, which cannot be ignored. The noise sources of these noises generally include thermal noise, scatter noise and dark current noise. Among them, thermal noise is a zero-mean white Gaussian random process and is the most important noise source. The effect of circuit noise at the receiving end, such as amplifier noise and dark current noise generated by diodes, is so small that it is negligible compared with thermal noise. Therefore, a variety of noise factors should be considered in threshold detection. We need a threshold that can be dynamically adjusted to follow changes in atmospheric noise to perform the signal mediation [3] [4].

The application of threshold detection technology in atmospheric laser communication has a wide application prospect. In 2001, Mukai et al. [5] of JPL laboratory in the United States first used adaptive threshold technology to improve communication performance in air ground optical communication link. He used adaptive threshold to estimate signal fading degree by average signal strength. In 2002, Bunis et al. [6] first introduced Kalman estimation into adaptive threshold estimation of atmospheric optical communication. In 2003, Zhu and Kahn of the University of California proposed using maximum likelihood sequence detection to reduce the loss of communication performance [7]. In 2004, Cole proposed to use the minimum mean square error to estimate the fading degree of signals. In weak turbulence, the detection performance of this algorithm is significantly higher than that of maximum likelihood algorithm [8]. In 2005, Namazi et al. [9] proposed binary data detection and synchronization method based on wavelet transform and adaptive Wiener filtering, and derived the calculation model of maximum optimization threshold. In 2009, Riediger proposed an algorithm that can significantly improve the detection performance of laser communication with long sequences: the sub-optimal sequence detection algorithm for maximum likelihood sequence detection, but this algorithm takes a long time to calculate.

In order to improve the communication quality, this paper draws on the research ideas of scholars, and on the basis of atmospheric turbulence model, the received signal is fading and noise is separated through the estimation of high-order cumulant, so as to realize the adaptive setting of signal decision threshold. 


\section{Theoretical Basis and Design Principle}

In the process of communication, in order to achieve the best reception, minimize the impact of noise on communication, and obtain the smallest bit error rate, we generally estimate the actual input value through a number of observed values, so as to be as close to the original signal as possible. Maximum Likelihood Estimate (MLE) is one of the most commonly used and effective estimation methods. First proposed by German mathematician C.F.Auss. Maximum likelihood estimation is a statistical method to find the parameters of the relevant probability density function of a sample set, which turns the probability density estimation problem into the parameter estimation problem.

In free space optical (FSO), the beam will undergo a series of changes due to the influence of atmospheric factors, among which atmospheric turbulence is an important reason for the random fluctuation of received optical signals. The following figure describes the principle of adaptive threshold design and implementation.

Figure 1 shows how the optimal adaptive threshold is implemented. In the figure, $r(n)$ is the received signal at the receiving end, $H_{0}$ and $H_{1}$ are the two cases of the maximum posterior probability receiver: The case where the transmitted character is 0 is recorded as $H_{0}$, and the case where the transmitted character is 1 is recorded as $H_{1} . Q_{0}$ and $Q_{1}$ are the process labeling quantities for threshold detection. Sampling each symbol of the received signal, the formed sampling matrix is divided into two operations: One way is to perform high-order cumulant operations on the sampling matrix to obtain estimated values of noise and turbulence fading related parameters, and the other way is to take the middle column of the sampling matrix as the symbol sequence to be detected. Then, input the estimated value of the noise parameter and the symbol sequence to be detected into the dashed box in the figure, calculate $H_{1}$ and $H_{0}$ under the assumption to get $Q_{0}$ and $Q_{1}$, and then $Q_{0}$ do exponential calculation, then multiply with the set initial value, this value is an initial reference value. The adaptive threshold value floats on this initial value as the turbulence changes, and the turbulence fading estimate participates in the averaging operation of the detection method. As the received signal changes continuously, the symbol sampling matrix is also changing, and the threshold will be updated in real time in the calculation of the adaptive algorithm along with the changing sampling matrix. When the value obtained is greater than 1 , the symbol to be detected is judged as $H_{1}$ the case (which is 1 ), when the value obtained is less than 1 , code element to be detected is judged as $H_{0}$ the case (which is 0 ). The adaptive decision is implemented by the following model [10]:

$$
\boldsymbol{r}=\frac{\gamma e \lambda}{h c} \boldsymbol{I}+\boldsymbol{n}_{1}+\boldsymbol{n}_{2}
$$

where $e$ represents the electron charge, $h$ is the Planck constant, $\lambda$ is the wavelength of the laser, $\gamma$ is the quantum efficiency of the photodetector, and $c$ is the speed of light in vacuum. Vector $\boldsymbol{r}$ is an electrical signal with an average 


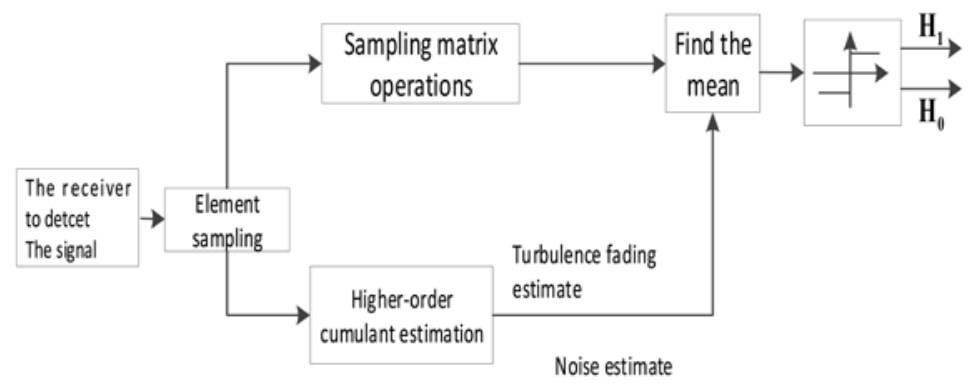

Figure 1. Principle of the optimal threshold.

level amplitude of $\mu$. The received signal $\boldsymbol{I}$ is the light intensity signal transmitted under strong turbulence, and its signal fading follows the turbulence distribution, which can be considered as the modulation of light intensity in time and space with the random variable of Gamma distribution. Vector $\boldsymbol{I}$ is used to represent the optical signal intensity sequence $I_{m}, I_{m}=x_{m} y_{m}$, matrix $\boldsymbol{M}_{y}$ is the covariance matrix of vector $y$. Among them, $x_{m}$ follows exponential distribution and $y_{m}$ follows Gamma distribution. Matrix $\boldsymbol{n}_{1}$ and matrix $\boldsymbol{n}_{2}$ are independent additive Gaussian noise terms, where matrix $\boldsymbol{n}_{1}$ represents independent additive zero-mean Gaussian noise independent of signal, such as thermal noise, amplifier noise, etc. Since these noises are independent of each other, the variance can be expressed as the sum of the variances of each noise. $\sigma_{n_{1}}^{2}=\sigma_{\text {total }}^{3}, \boldsymbol{n}_{1} \in N\left(0, \sigma_{n_{1}}^{2} \boldsymbol{E}\right)$. Matrix $\boldsymbol{n}_{2}$ represents the avalanche diode noise. Proportional to the current signal, when receiving high and low level "1" and "0", $\sigma_{n_{2}}^{2}$ means $\sigma_{n_{2} L}^{2}$ and $\sigma_{n_{2} H}^{2}$ respectively, $n_{2} \in N\left(0, \sigma_{n_{2}}^{2} \boldsymbol{M}_{n_{2}}\right)$. According to the maximum posterior probability criterion, the best decision formula of the transmitted character is obtained [11]:

$$
\hat{s}=\arg \max P(r \mid s) P(s)
$$

In the formula, the case where the transmitted character $s$ is 0 is recorded as $H_{0}$. The case where the transmitted character $s$ is 1 is recorded as $H_{1}$. When in $H_{0}$ case. The transmitted signal is only noise, so $r(t)=n(t)$. There is no turbulent decline. Therefore, the probability density function of $r(t)$ can be expressed as [12]:

$$
f_{r}\left(\boldsymbol{r} \mid H_{0}\right) \int_{0}^{\infty} \frac{1}{(2 \pi)^{M}} \exp \left[-\frac{1}{2} \boldsymbol{r}^{H}\left(\sigma_{n_{1}}^{2} \boldsymbol{E}+\sigma_{n_{2}}^{2} \boldsymbol{M}_{n_{2}}\right)^{-1} \boldsymbol{r}\right]
$$

And when in $H_{1}$ case, the probability density function of $r(t)$ is expressed as:

$$
\begin{aligned}
& f_{r}\left(\boldsymbol{r} \mid H_{1}\right) \int_{0}^{\infty} \frac{1}{(2 \pi)^{M}\left|x \boldsymbol{M}_{y}+\sigma_{n_{1}}^{2} \boldsymbol{E}+\sigma_{n_{2}}^{2} \boldsymbol{M}_{n_{2}}\right|} \\
& \times \exp \left[-\frac{1}{2} \boldsymbol{r}^{H}\left(x \boldsymbol{M}_{y}+\sigma_{n_{1}}^{2} \boldsymbol{E}+\sigma_{n_{2}}^{2} \boldsymbol{M}_{n_{2}}\right)^{-1} \boldsymbol{r}\right] f_{x}(x) \mathrm{d} x
\end{aligned}
$$

In Equation (3) and Equation (4), the vector $\boldsymbol{r}$ in the formula represents the received signal sequence, $f_{r}$ is the probability density function of the received signal $r(t), \sigma_{n_{1}}^{2}$ is the Gaussian noise variance independent of signal, $\sigma_{n_{2}}^{2}$ is 
the noise variance related to the received signal current. Matrix $\boldsymbol{M}_{y}$ is the covariance matrix of vector $\boldsymbol{y}$, matrix $\boldsymbol{M}_{n_{2}}$ is the covariance matrix of the Gaussian process related to the received signal, matrix $\boldsymbol{E}$ is the identity matrix, probability density function of $f_{x}(x)$, make:

$$
\left\{\begin{array}{l}
Q_{0}=-\frac{1}{2} \boldsymbol{r}^{H}\left(\sigma_{n_{1}}^{2} \boldsymbol{E}+\sigma_{n_{2}}^{2} \boldsymbol{M}_{n_{2}}\right)^{-1} \boldsymbol{r} \\
Q_{1}=-\frac{1}{2} \boldsymbol{r}^{H}\left(x \boldsymbol{M}_{y}+\sigma_{n_{1}}^{2} \boldsymbol{E}+\sigma_{n_{2}}^{2} \boldsymbol{M}_{n_{2}}\right)^{-1} \boldsymbol{r}
\end{array}\right.
$$

$Q_{0}$ and $Q_{1}$ are the mark amount of the calculation process, which has no practical meaning. According to the maximal posterior probability minimum error criterion [13]:

$$
\int_{0}^{\infty} \frac{\exp Q_{1}-\xi \cdot \exp Q_{0}}{\left|x \boldsymbol{M}_{y}+\sigma_{n_{1}}^{2} \boldsymbol{E}+\sigma_{n_{1}}^{2} \boldsymbol{M}_{n_{2}}\right|} f_{x}(x) \mathrm{d} x \underset{H_{0}}{\stackrel{H_{1}}{>} \frac{P\left(H_{1}\right)}{P\left(H_{0}\right)}}
$$

Suppose the signal is sent with equal probability of 1 and $0, \frac{P\left(H_{1}\right)}{P\left(H_{2}\right)}=1$, according to Neiman-Pearson basic principles [14], $\xi$ is the normalized threshold. The judgment condition is $\frac{\Lambda(r)}{\zeta}>_{H_{0}}^{\mathrm{H}_{1}} 1$, get the formula to determine the threshold as [15]:

$$
\int_{0}^{\infty} \frac{\exp Q_{1}-\xi \cdot \exp Q_{0}}{\left|x \boldsymbol{M}_{y}+\sigma_{n_{1}}^{2} \boldsymbol{E}+\sigma_{n_{1}}^{2} \boldsymbol{M}_{n_{2}}\right|} f_{x}(x) \mathrm{d} x \underset{H_{0}}{\stackrel{H_{1}}{>}} 1
$$

From Equation (7), it can be seen that the adaptive parameters that need to be estimated include the mean value $\mu$ of the signal and the order parameter $v$. Covariance matrix $\boldsymbol{M}_{y}$ of distributed fading, Gaussian noise related parameters $\sigma_{n_{1}}^{2}, \sigma_{n_{2}}^{2}$ and matrix $\boldsymbol{M}_{n_{2}}$.

To predict the threshold determined by Equation (7), it is necessary to estimate the high-order cumulant for turbulent fading, and estimate the second-order cumulant for Gaussian noise, and deal with signal fading and noise separately. You can set $\frac{\gamma e \lambda}{h c}=1$, the signal sequence detected by the avalanche diode at the receiving end of the atmospheric laser communication system as:

$$
r(n)=x(n) y(n)+v_{1}(n)+v_{2}(n),(n=1, \cdots, m)
$$

Sequence $x(n)$ obeys exponential distribution, sequence $y(n)$ obeys Gamma distribution, $v_{1}(n)$ and $v_{2}(n)$ are Gaaussian noise independent of the signal and avalanche diode receiving current related noise sampling sequence.

\section{High Order Cumulant Estimation}

There will be turbulence fading in the atmospheric turbulence channel. Here we estimate the high-order cumulant of turbulence fading, and estimate the second 
moment of Gaussian noise on this basis. Use the protection characteristics between the high-order cumulant and the low-order cumulant to analyze the high-order cumulant of the innovation process obtained from the light intensity signal sequence. In order to reduce the computational complexity, the parameters of the turbulent channel and the mean value of the Gamma parameter are jointly estimated by the fourth-order and sixth-order cumulants with zero delay.

When $k>2$, the k-order cumulant of Gaussian noise is 0 . The second-order cumulant of $r(n)$ is equal to the autocorrelation function as:

$$
c_{2}^{r}[l]=E\left[r(i) r^{*}(i+l)\right]=m_{2}^{x}[l] m_{2}^{y}[l]+m_{2}^{v}[l]
$$

$v_{1}(n)$ and $v_{2}(n)$ are two Gaussian random processes with zero mean. For Gaussian random process, when the order is greater than 2, its cumulant is zero, that is to say, the higher-order cumulant of gaussian process is always zero. So, the third-order cumulant of $r(n)$ as:

$$
\begin{aligned}
c_{3}^{r}\left[l_{1}, l_{2}\right] & =m_{3}^{r}\left[l_{1}, l_{2}\right]=E\left\{\left[r(n) r^{*}\left(n+l_{1}\right) r\left(n+l_{2}\right)\right]\right\} \\
& =m_{3}^{I}\left[l_{1}, l_{2}\right]=m_{3}^{x}\left[l_{1}, l_{2}\right] m_{3}^{y}\left[l_{1}, l_{2}\right]=0
\end{aligned}
$$

The relationship between the fourth-order cumulant and the fourth-order moment and the second-order moment as:

$$
\begin{aligned}
c_{4}^{r}\left[l_{1}, l_{2}, l_{3}\right]= & \left(m_{4}^{x}\left[l_{1}, l_{2}, l_{3}\right]-m_{2}^{x}\left[l_{1}\right] m_{2}^{x}\left[l_{3}-l_{2}\right]\right) \cdot m_{2}^{y}\left[l_{1}\right] m_{2}^{y}\left[l_{3}-l_{2}\right] \\
& +\left(m_{4}^{x}\left[l_{1}, l_{2}, l_{3}\right]-m_{2}^{x}\left[l_{3}\right] m_{2}^{x}\left[l_{1}-l_{2}\right]\right) \cdot m_{2}^{y}\left[l_{3}\right] m_{2}^{y}\left[l_{1}-l_{2}\right]
\end{aligned}
$$

Estimate $m$ becomes a key issue, the relationship of higher-order cumulants can be written as:

$$
c_{2}^{I}\left[l_{1}\right]=m_{2}^{x}\left[l_{1}\right] c_{2}^{y}\left[l_{1}\right]=k \sum_{l_{2}=-\infty}^{+\infty} \sum_{l_{3}=-\infty}^{+\infty} c_{2}^{y}\left[l_{1}, l_{2}, l_{3}\right]
$$

where $k$ is the correlation coefficient. From formula (12), we know if get $c_{4}^{r}\left[l_{1}, l_{2}, l_{3}\right]$, need to calculate the cumulant of all time domain delay, the computation is too large, so the redundant time needs to be eliminated. Because the fourth order cumulant has the following redundant relation:

$$
c_{4}^{r}\left[l_{1}, l_{2}, l_{3}\right]=c_{4}^{r}\left[l_{3}, l_{2}, l_{1}\right]=c_{4}^{r}\left[l_{3}-l_{2},-l_{2}, l_{1}-l_{2}\right]=c_{4}^{r}\left[l_{1}-l_{2},-l_{2}, l_{3}-l_{2}\right]
$$

Consider the zero delay cumulant, $l_{1}=l_{2}=l_{3}=0$. According to the definition of the high-order origin moment, it can be known that $m_{2}^{y}[0]=2$, work out :

$$
c_{4}^{r}[0,0,0]=2\left\{m_{4}^{x}[0,0,0]-\left(m_{2}^{x}[0]\right)^{2}\right\}\left(m_{2}^{y}[0]\right)^{2}=\frac{8 \mu^{4}}{v}\left(1+\frac{1}{v}\right)\left(\frac{4}{v}+\frac{6}{v}\right)
$$

In the formula, the order parameter $v$ and the mean value $\mu$ are unknown in practice, and a high-order cumulant with zero delay is required for joint estimation. Estimate $m_{2}^{y}[0]=2$ based on the non-redundant time domain.

\subsection{Estimation of Order Parameter and Mean Value of Atmospheric Turbulence Distribution}

For turbulence distribution order parameter $v$ and estimate of mean $\mu$, 
the sixth-order cumulant of the signal as [15]:

$$
\begin{aligned}
& c_{6}^{r}\left[\tau_{1}, \tau_{2}, \tau_{3}, \tau_{4}, \tau_{5}\right] \\
& \triangleq \operatorname{cum}\left\{r[n], r^{*}\left[n+\tau_{1}\right], r\left[n+\tau_{2}\right], r^{*}\left[n+\tau_{3}\right], r\left[n+\tau_{4}\right], r^{*}\left[n+\tau_{5}\right]\right\}
\end{aligned}
$$

The second, fourth, and sixth moments of the Gamma distribution as:

$$
\left\{\begin{array}{l}
E\left\{x^{2}\right\}=\mu^{2}\left(1+\frac{1}{v}\right) \\
E\left\{x^{4}\right\}=\mu^{4}\left(1+\frac{1}{v}\right)\left(1+\frac{2}{v}\right)\left(1+\frac{3}{v}\right) \\
E\left\{x^{6}\right\}=\mu^{6}\left(1+\frac{1}{v}\right)\left(1+\frac{2}{v}\right)\left(1+\frac{3}{v}\right)\left(1+\frac{4}{v}\right)\left(1+\frac{5}{v}\right)
\end{array}\right.
$$

$y$ obey the exponential distribution, so $E\left\{y^{k}\right\}=k !$. Get:

$$
\left\{\begin{array}{l}
c_{6}^{r}[0,0,0,0,0]=\mu^{6}\left(\frac{384 v^{5}+8064 v^{4} 54144 v^{3}}{v^{5}}+\frac{154752 v^{2}+194688 v+86400}{v^{5}}\right) \\
c_{4}^{r}[0,0,0]=4 \mu^{4} \frac{4 v^{4}+10 v+6}{v^{3}}
\end{array}\right.
$$

Moment estimators $\hat{m}_{2}^{r}[0], \quad \hat{m}_{4}^{r}[0,0,0]$ and $\hat{m}_{6}^{r}[0,0,0,0,0]$ are respectively represented by $m$ sampling mean values:

$$
\left\{\begin{array}{l}
\hat{m}_{2}^{r}[0]=\frac{1}{m K} \sum_{K=1}^{k} \sum_{n=1}^{m}\left|z_{k}(n)\right|^{2} \\
\hat{m}_{4}^{r}[0,0,0]=\frac{1}{m K} \sum_{K=1}^{k} \sum_{n=1}^{m}\left|z_{k}(n)\right|^{4} \\
\hat{m}_{6}^{r}[0,0,0]=\frac{1}{m K} \sum_{K=1}^{k} \sum_{n=1}^{m}\left|z_{k}(n)\right|^{6}
\end{array}\right.
$$

From this, we can get estimates of cumulants $c_{6}^{r}[0,0,0,0,0]$ and $c_{4}^{r}[0,0,0]$, Simultaneous equations estimate $\hat{\mu}$ and $\hat{v}$ :

$$
\left\{\begin{array}{l}
\hat{\mu}=\frac{3}{2} \frac{\left(\hat{c}_{4}^{r}[0,0,0]\right)^{2}}{\hat{c}_{6}^{r}[0,0,0,0,0]} \\
\hat{\mu}=\frac{18\left(\hat{c}_{4}^{r}[0,0,0]\right)^{3}}{\left(\hat{c}_{6}^{r}[0,0,0,0,0]\right)^{2}}
\end{array}\right.
$$

\subsection{Estimation of Second and Fourth Order Cumulants}

According to the above definition and analysis, the following relationship is obtained: the following relationships are obtained:

$$
\hat{c}_{4}^{r}[l, l, l]=\hat{m}_{4}^{r}[l, l, l]-2 \hat{m}_{2}^{r}[0] \hat{m}_{2}^{r}[l]
$$

According to Taylor's freezing theory [16], the turbulence change speed is negligible compared to the signal transmission speed Suppose the signal sequence is $z(n)$, express it as:

$$
z_{k}[n]=r_{k}[n]=x_{k}[n] y_{k}[n]+v_{1 k}[n]+v_{2 k}[n], k=1,2, \cdots, K
$$


Sample $m$ times in one symbol period, the $m$-th symbol sampling signal is recorded as $\left\{z_{k}[n] ; n=1, \cdots, m\right\}$. And arrange the entire $m$ sample value on vector $\mathbf{z}_{k}$, can calculate moment estimates $m_{2}^{r}[l]$ and $m_{4}^{r}[l, l, l]$ :

$$
\begin{gathered}
m_{2}^{r}[l]=\frac{1}{m-l} \sum_{n=1}^{m-l}\left(\frac{1}{K} \sum_{K=1}^{K} \operatorname{Re}\left\{z_{k}[n] z_{k}^{*}[n+l]\right\}\right) \\
m_{4}^{r}[l, l, l]=\frac{1}{m-l} \sum_{n=1}^{m-l}\left(\frac{1}{K} \sum_{K=1}^{K} \operatorname{Re}\left\{z_{k}[n+l] z_{k}^{*}[n+l]^{2}\right\}\right)
\end{gathered}
$$

where $l=0, \cdots, m-l$. From Equations (22) and (23), the average of $m-l$ estimators obtained for each signal sequence $n$, get an estimate of $C_{4}^{r}[l, l, l]$.

Sample $m$ in each symbol period, store the data of $K$ symbols in matrix $z_{k}[n]$, use Equations (22) and (23) to calculate the second and fourth moments, and then substitute Equation (20) to calculate The fourth-order cumulant is calculated.

\section{Simulation Results}

Set different mean value $\mu$ and order parameter $v$. According to the distribution function, use Monte Carlo method to generate the distribution test signal sequence. The specific sizes of $\mu$ and $v$ are selected according to the specific requirements of each simulation below, given in the simulation below, the generated test sequence is $r(n)$. After rearranging $r(n)$, the threshold algorithm updated in real time is obtained. Under OOK modulation, the bit error rate of the defined distribution is

$$
S E R=\frac{E\left[r_{n}^{2}\right]}{E\left[\hat{r}_{n, n+\tau}^{2}\right]}
$$

Define the signal-to-noise ratio is

$$
S N R=\frac{E\left[I^{2}\right]}{2 \sigma_{n}^{2}}=\frac{\mu}{2 \sigma_{n}^{2}}
$$

Figure 2 shows the relationship between the delay $l$ and the second-order moment estimation value. When the test sequence is generated, the order parameter and the mean value are given, and the theoretical values of other parameters can be calculated. When the test symbol changes from 64 to 10,000 , the test symbol The more, when the test sequence is generated, the estimated value of the theoretical second moment is closer to the theoretical value. Therefore, the number of test symbols can be increased and the performance of the adaptive threshold strategy can be improved.

\section{Change of Bit Error Rate with Signal to Noise Ratio}

Simulation initial conditions: sampling at $50 \%$ of the pulse of the received digital signal, comparing the sampled value with a fixed threshold, determine whether the digital signal is " 0 " or " 1 ", make mean value is $\mu=1$, order parameter is $v=2$, the fixed threshold is 0.5 . 


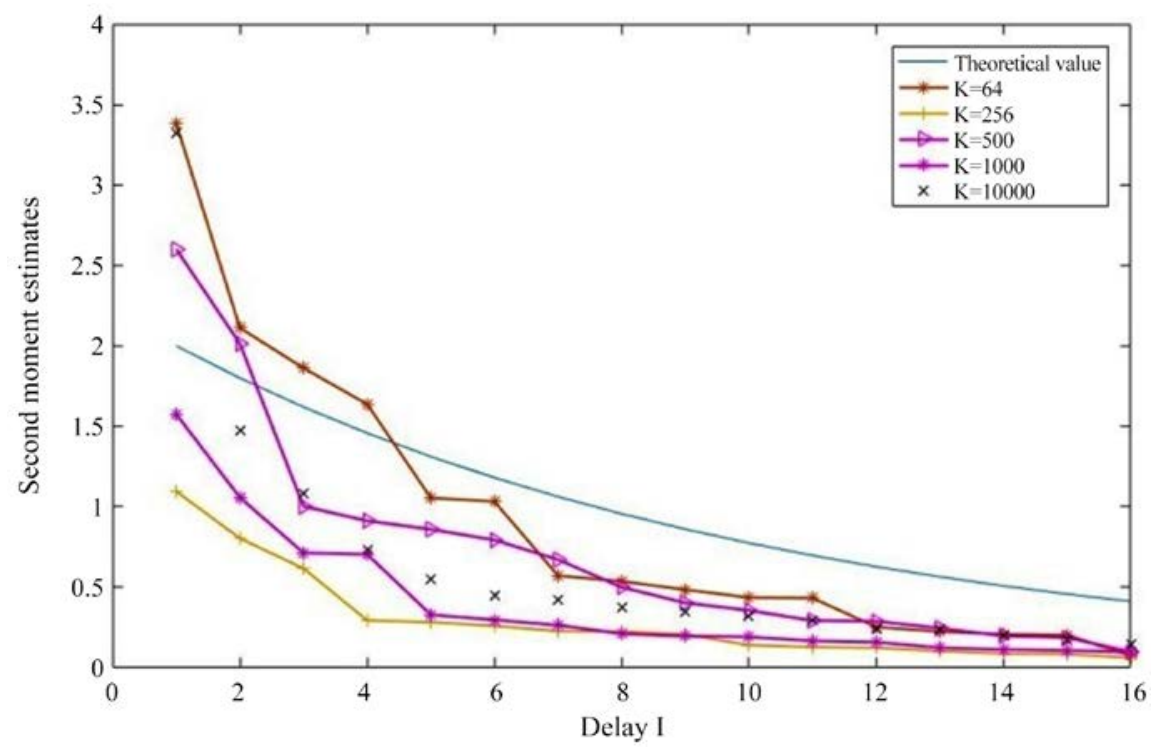

Figure 2. Estimated $m_{2}^{v}[l]$ sequence.

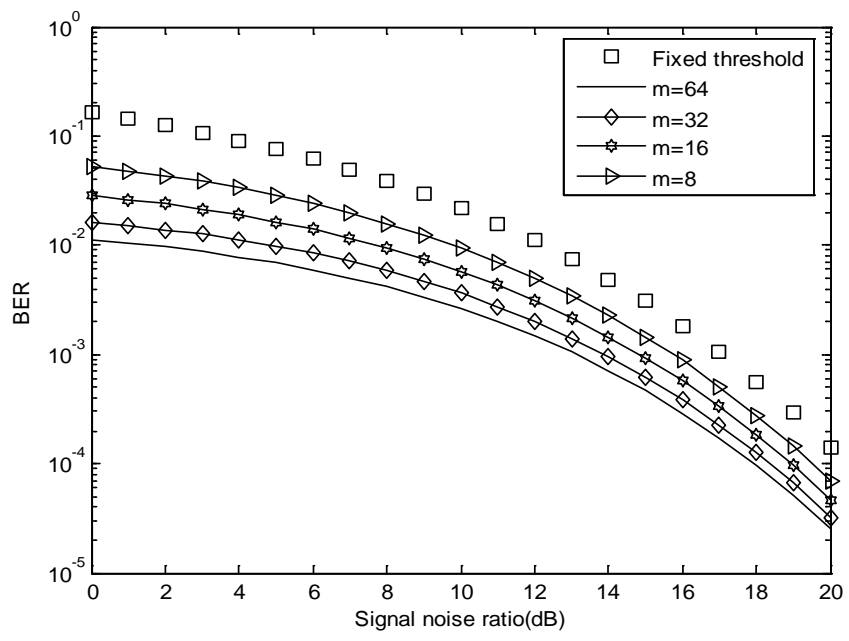

Figure 3. Time slot error rate curve along with the change of signal noise ratio.

Figure 3 shows the variation curve of the bit error rate with the signal-to-noise ratio of the fixed threshold detection method and the adaptive threshold detection method. It can be seen that when the signal-to-noise ratio is the same, the bit error rate of the received signal of the adaptive threshold detection method is significantly lower than that of the fixed threshold detection method, indicating that the adaptive detection method improves system performance better than the fixed threshold detection method.

\section{Conclusions}

This paper discusses the adaptive threshold detection model and analyzes its performance for the signal detection problem of atmospheric turbulence channel. 
Compared with the fixed threshold detection method, the method in this paper can significantly reduce the bit error rate of the communication system; the larger the number of symbol samples, the better the system performance, but the more complex the algorithm is. In order to reduce the computational complexity, the order parameters and the mean values of the turbulence gamma parameters are jointly estimated by the fourth and sixth order cumulants with zero delay. The results show that the method can suppress the influence of strong turbulence on the received signal of laser communication system and improve the communication quality of the system. The above data results show that the application of adaptive threshold in atmospheric laser communication is feasible. This paper studies the implementation of adaptive threshold under normal conditions, but in actual atmospheric communication, turbulence changes randomly. Therefore, in future research, we can study the implementation of adaptive threshold separately under different turbulence conditions. It is also necessary to study how to ensure the smooth transition of the respective adaptive schemes when the turbulence of different intensities changes without affecting the normal communication.

At present, although there are still some technical problems to be improved in atmospheric laser communication, the unique advantages and broad market fields of atmospheric laser communication have huge potential. I believe that with the continuous efforts of many scientific scholars, the atmospheric laser communication will be more stable and reliable in the future, and it will play an increasingly important role in life.

\section{Acknowledgements}

This work was supported by Key industry innovation chain project of Shaanxi Province (2017ZDCXL-GY-06-01), Scientific Research Program of Education Department of Shaanxi Province (18JK0341), and Xi'an Science and Technology Innovation Guidance Project (201805030YD8CG14(12)).

\section{Conflicts of Interest}

The authors declare no conflicts of interest regarding the publication of this paper.

\section{References}

[1] Wang L.L. and Ke, X.Z. (2004) Research and Simulation on Effect of Turbulence to Optical Communication. The Journal of Light Scattering, 16, 250-255.

[2] Uysal, M., Navidpour, S.M. and Li, J. (2004) Error Rate Performance of Coded Free-Space Optical Links Over Strong Turbulence Channels. IEEE Communications Letters, 8, 635-637. https://doi.org/10.1109/LCOMM.2004.835306

[3] Wang, J., Huang, D.X. and Yuan, X.H. (2007) Adaptive Signal Reception Based on HOS Cumulants in Optical Wireless Communication System over Strong Turbulence. Acta Photonica Sinica, 36, 1078-1082.

[4] Chen, C.Y. and Yang, H.M. (2009) Research Progress of Mitigation Technologies of 
Turbulence Effects in Atmospheric Optical Communication. Acta Armamentarii, 6, 779-791.

[5] Mukai, R., Arabshahi, P. and Yan, T.Y. (2001) An Adaptive Threshold Detector and Channel Parameter Estimator for Deep Space Optical Communications. IEEE Global Telecommunications Conference, 1, 50-54.

https://doi.org/10.1109/GLOCOM.2001.965078

[6] Burris, H.R., Reed, A.E., et al. (2002) Adaptive Thresholding for Free-Space Optical Communication Receivers with Muliplicative Noise. IEEE Aerospace Conference Proceedings, Big Sky, MT, 2002, 3.

[7] Zhu X.M., Kahn, J.M. and Wang, J. (2003) Mitigation of Turbulence-Induced Scintillation Noise in Free-Space Optical Links Using Temporal-Domain Detection Techniques. IEEE Photonics Technology Letters, 15, 623-625.

https://doi.org/10.1109/LPT.2003.809305

[8] Cole, M. and Kiasaleh, K. (2004) Signal Intensity Estimators for Free-Space Optical Communications through Turbulent Atmosphere. IEEE Photonics. Technology Letters, 16, 2395-2397. https://doi.org/10.1109/LPT.2004.833924

[9] Namazi, N., Burris, R. and Charmaine Gilbreath, G. (2005) Analytical Approach to Calculation of Probability of Bit Error and Optimum Thresholds in Free-Space Optical Communication. Optical Engineering.

[10] Chen, C.Y. and Yang, H.M. (2009) Research Progress of Mitigation Technologies of Turbulence Effects in Atmospheric Optical Communication. Acta Armamentarii, 30, 779-791.

[11] Lee, P.J. (1986) Computation of the Bit Error Rate of Coherent M-ary PSK with Gray Code Bit Mapping. IEEE Transactions on Communications, 34, 488-491. https://doi.org/10.1109/TCOM.1986.1096558

[12] Dantzig, G.B. and Wald, A. (1951) On the Fundamental Lemma of Neyman and Pearson. The Annals of Mathematical Statistics, 22, 87-93. https://doi.org/10.1214/aoms/1177729695

[13] Watts, S. (1987) Radar Detection Prediction in K-distributed Sea Clutter and Thermal Noise. IEEE Transactions on Aerospace and Electronic Systems, 23, 40-45. https://doi.org/10.1109/TAES.1987.313334

[14] Gini, F. (1997) A Cumulant-Based Adaptive Technique for Coherent Radar Detection in a Mixture of K-Distributed Clutter and Gaussian Disturbance. IEEE Transactions on Signal Processing, 45, 1507-1519. https://doi.org/10.1109/78.599993

[15] Zhang, X.D. (1994) Modern Signal Processing. Tsinghua University Press, BeiJing, 21-203.

[16] Farina, A. and Russo, A. (1986) Radar Detection of Correlated Targets in Clutter. IEEE Transactions on Aerospace and Electronic Systems, 22, 513-532. https://doi.org/10.1109/TAES.1986.310717 